\title{
Cross Platform Application for Smart Address Book
}

\author{
Bhushan S. Thakare \\ Assistant Professor \\ Department of Computer \\ Engineering, \\ Sinhgad Academy of \\ Engineering, \\ Pune, India.
}

\author{
Dhanashree Shirodkar \\ Department of Computer \\ Engineering, \\ Sinhgad Academy of \\ Engineering, \\ Pune, India.
}

\author{
Naghma Parween \\ Department of Computer Engineering, \\ Sinhgad Academy of Engineering, \\ Pune, India.
}

\author{
Shama Parween \\ Department of Computer \\ Engineering, \\ Sinhgad Academy of \\ Engineering, \\ Pune, India.
}

\begin{abstract}
Address Book allows users to enter all sorts of information about a person, including phone number, address, email id, etc. If someone's phone number or address gets changed then they need to inform to all their friends about new address/phone number. Generally we use facebook/whatsapp or do SMS to all friends to update our number/address. Here our application aims to update contact information automatically in contact book of users by maintaining profile of all users to make any update. Also an additional feature of locating your nearest friend using GPS is also available so in case of any emergency they can find who is nearest to them and can contact them right away.

This application is developed keeping different smart phone users in mind. So with the help of PhoneGap, we have developed a cross platform application which can run on most of the operating systems like android, iOS, windows etc.
\end{abstract}

\section{Keywords}

Cross platform; smart phones; Address book; GPS.

\section{INTRODUCTION}

Smart phones have brought a new era of mobiles and tablets. The market of smart phones is growing rapidly with new operating systems coming into picture along with the already existing ones. Making a new application that can run on all the operating system is difficult and costly as they are written in their native language. A cross-platform application is an easy solution. Using PhoneGap we have developed a cross platform application for automating the task of updating address book.

Address book is a database that stores essential information like name, contact number, address, e-mail id etc of our friends, family and other important people. If someone's address gets changed then they need to inform to their friends about new address/phone number. Generally we use facebook/whatsapp or do SMS to all friends to update our number/address. Here our application will update contact/address of our smart phone if somebody changed his/her contact information. The application maintains profile of all users to make any updates. Also the application contains feature of location tracking. Location of the users using this app can be checked. Other features like privacy for contact, profile photo, location are also present in this app.
The app being cross platform can be used on various mobile operating system such android OS, windows OS etc. The languages used to develop the cross platform app are HTML5, PHP and JavaScript.

\section{ALGORITHM}

The application is a client-server type application. Client will run on mobile handset and will communicate with server for making any update. Client will ask user to create his/her profile, it will forward that information to server to get added in database. Client will also ping server every after some time to check if any contact needs update, if yes then it will fetch information form server and update the contact information accordingly.

Server sends all data coming from different clients in to database using BASE64 encryption algorithm. Server will also check the user policy so that it will inform to those users only which are part of policy.

There will be user \& group level policy to be used so that a user can decide to whom server should send the contact update information. In any user selects only 2 of its friend should get her/his contact update then server will send update information to only those two friends.

This product will be using web services on server side. REST web services are built to work best on the Web. Representational State Transfer (REST) is an architectural style that specifies constraints, such as the uniform interface, that if applied to a web service induces desirable properties, such as performance, scalability, and modifiability that enable services to work best on the Web. In the REST architectural style, data and functionality are considered resources and are accessed using Uniform Resource Identifiers (URIs), typically links on the Web. The resources are acted upon by using a set of simple, well-defined operations. The REST architectural style constrains architecture to client/server architecture and is designed to use a stateless communication protocol, typically HTTP. In the REST architecture style, clients and servers exchange representations of resources by using a standardized interface and protocol. 


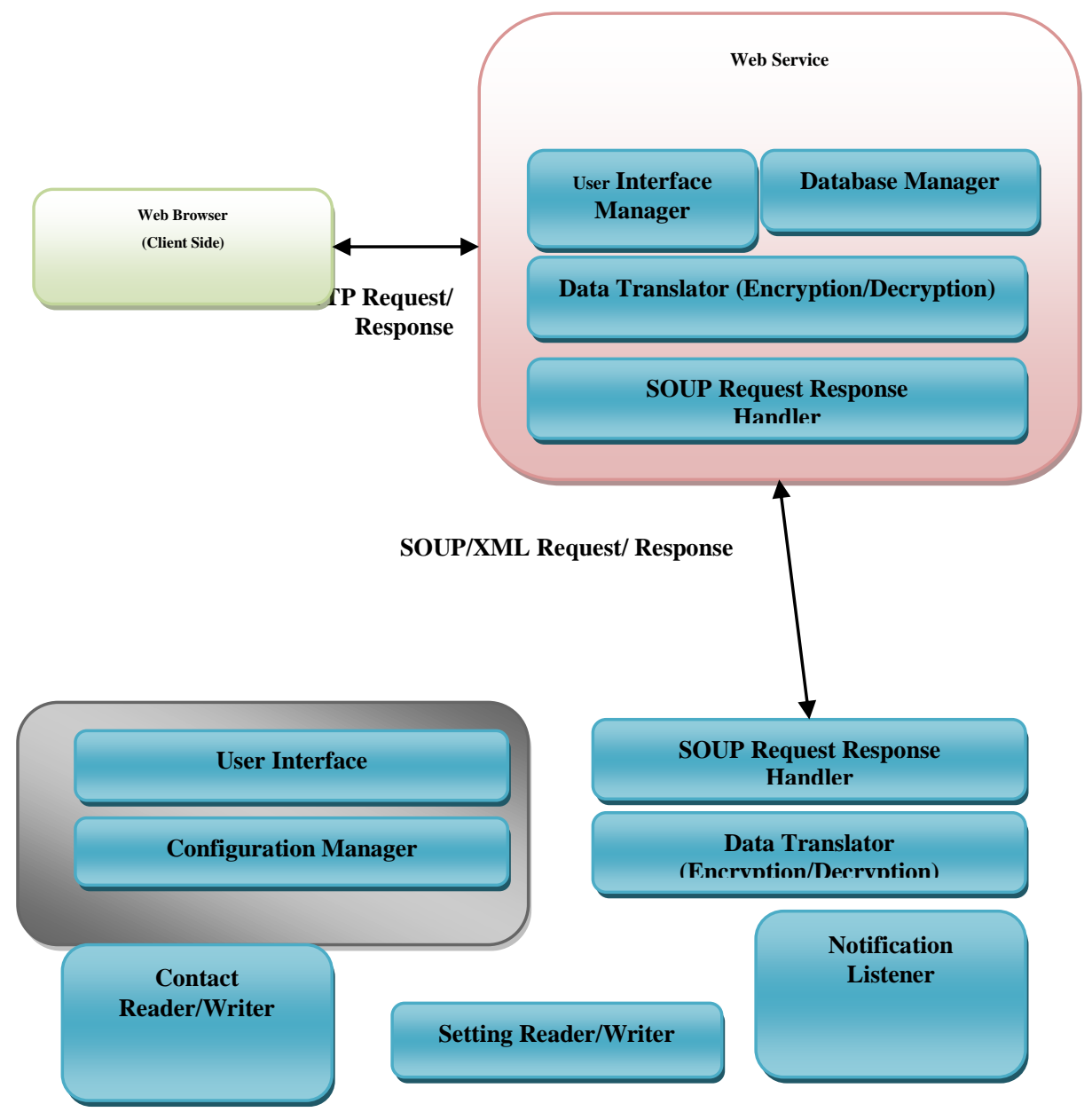

There are two algorithms which will be used in this project. They are:

Base 64 algorithm

Algorithm for distance calculation

\section{Base 64 Algorithm}

Server will send all data coming from different clients in to database using BASE64 encryption algorithm.

Base64 is a group of similar binary-to-text encoding schemes that represent binary data in an ASCII string format by translating it into a radix-64 representation.

Base64 encoding schemes are commonly used when there is a need to encode binary data that needs to be stored and transferred over media that are designed to deal with textual data. This is to ensure that the data remains intact without modification during transport.

The Base64 encoding process is to:

Divide the input bytes stream into blocks of 3 bytes.

Divide 24 bits of each 3-byte block into 4 groups of 6 bits.

Map each group of 6 bits to 1 printable character, based on the 6-bit value using the Base64 character set map.

If the last 3-byte block has only 1 byte of input data, pad 2 bytes of zero ( $\mid \mathrm{x} 0000)$. After encoding it as a normal block, override the last 2 characters with 2 equal signs (==), so the decoding process knows 2 bytes of zero were padded.

If the last 3 -byte block has only 2 bytes of input data, pad 1 byte of zero ( $(\mathrm{x} 00)$. After encoding it as a normal block, override the last 1 character with 1 equal signs $(=)$, so the decoding process knows 1 byte of zero was padded.

Carriage return (\r) and new line ( $\backslash n)$ are inserted into the output character stream. They will be ignored by the decoding process.

Input data, 3 bytes, "ABC". Encoded output, 4 characters, "QUJD"

Input Data A B C

Input Bits 010000010100001001000011

$$
111
$$

Bit Groups 010000010100001001000011

$$
\text { Mapping Q U J D }
$$

Use of Phonegap

The demand for cross platform mobile development is constantly growing in the market. The basic need for programmers on any platform is compatibility. There are various cross-browser tools that will take any developer a long way in creating flexible apps that stand the compatibility test such as Whoop, Mosync, Phonegap, Appcelator etc. We are developing this application with the help of Phonegap. 
PhoneGap applications are developed using HTML, CSS, and JavaScript, however the final product of a PhoneGap application is a binary application archive that can be distributed through standard application ecosystems. For iOS applications the output is an IPA file) (iOS Application Archive), for Android applications the output is an APK file) (Android Package), for Window Phone the output is a XAP file (Application Package), etc.. These are the same application packaging formats used by "native" applications, and can be distributed through the appropriate ecosystems (iTunes Store, Google Play, Amazon Market, BlackBerry App World, Windows Phone Marketplace, etc.)

\section{EXPERIMENTAL RESULTS}

The application runs on a chrome less browser. It selects the default browser of user's smart phone and launches the application in that browser. Since we are using phone gap, we had to select one operating system at first to run and test our application. We selected Android OS as our base for this application development. We also needed to specify a PhoneGap version for our project and accordingly we could get access to different API's of Android OS. Our application is supported by Android 4.0 and above versions only.

Our application needs to connect to the server first; to connect to the server the client needs the IP address of the server to identify the server on the network. The application contains section for sign up for new users and login. A valid user has 3 options, to set profile, select privacy and view map. The first two options are linked to our main application i.e. to update contact. In set profile the user first fills details about themselves and saves it. This detail is saved into database by the server. Also, select privacy enables user to filter down only those contacts whom you wish to send updated number.

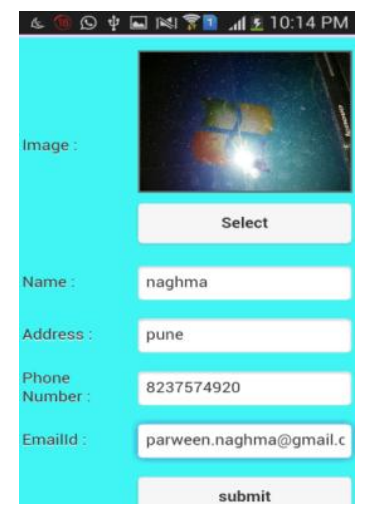

Fig. Profile page

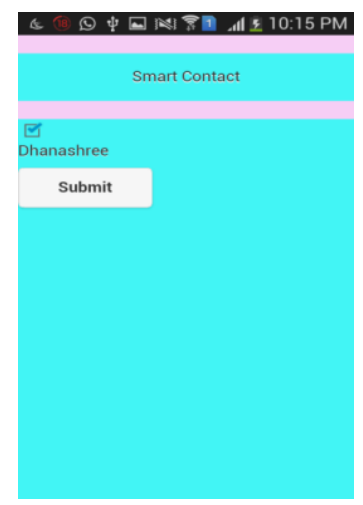

Fig. 3.2 Select privacy page
If you make any changes in the set profile page and save it, it will be registered by the server and the contact book of the selected contact's contact book will be updated automatically.

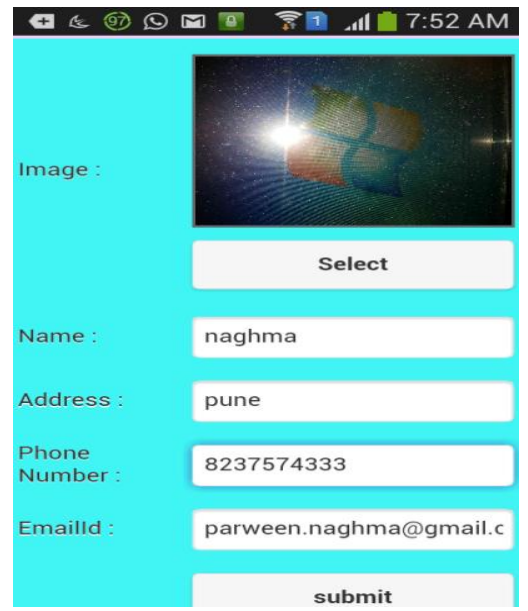

Fig. 3.3Changing Phone number

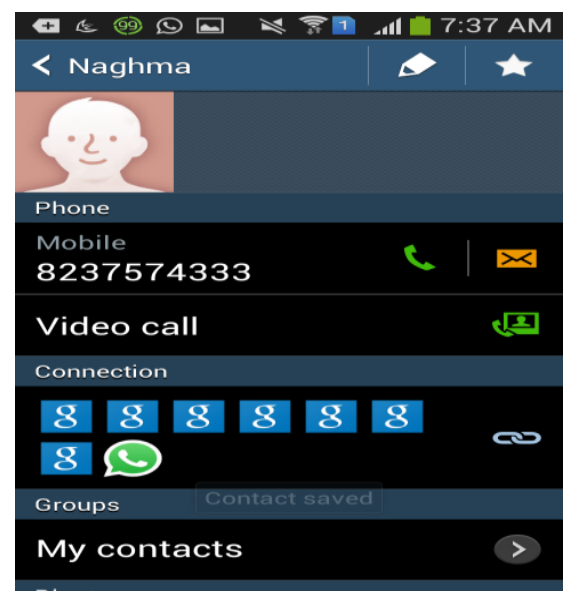

Fig. 3.4 Updated Contact

The view map section integrates Google maps and requires internet connection. User's location is obtained using GPS. It shows location of user's friends along with user.

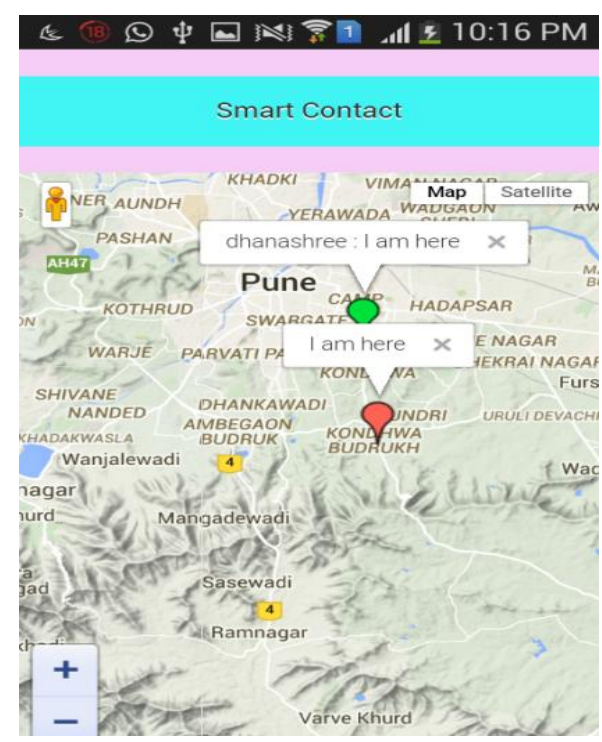

Fig.3.5 View Map page 


\section{CONCLUSION}

The main aim of our project was to automate the task of updating contact book of smart phone users. The increasing number of mobile platforms restricted us from developing native mobile apps for even the major operating systems as it is cost effective and time consuming. So we used cross platform approach for our project using PhoneGap. Although cross platform approach has lot of limitation, our project implemented successfully. Along with updating contact book, additional feature of finding nearby friends using GPS can be done by using our application. Although the look and feel of native application is much better than any cross platform application, our application stands in term of heterogeneity, scalability and performance over cost.

\section{REFERENCES}

[1] Stelios Xinogalos and Spyros Xanthopoulos,"A Comparative Analysis of Cross-platform Development Approaches for Mobile Applications “

[2] "State of art approaches to build cross platform mobile application" Vol 107 No. 20 by IJCA Journal 2014

[3] "Cross Platform Application Development", http://www.ndot.in/cross-platform-mobiledevelopment.html

[4] "state of art approaches using phonegap for portability of mobile apps" IJARCCE Journal 2014.

[5] About PhoneGap, "How PhoneGapWorks", http://phonegap.com/about 